\title{
Insulin pump basal adjustment for exercise in type 1 diabetes: a randomised crossover study
}

\author{
Sybil A. McAuley ${ }^{1,2}$ • Jodie C. Horsburgh ${ }^{1}$ - Glenn M. Ward ${ }^{2,3}$ • André La Gerche ${ }^{1,4,6}$. \\ Judith L. Gooley $^{1}$ - Alicia J. Jenkins ${ }^{1,2,5}$ • Richard J. MacIsaac ${ }^{1,2}$ • David N. O'Neal ${ }^{1,2}$
}

Received: 29 January 2016 / Accepted: 29 March 2016 / Published online: 11 May 2016

(C) Springer-Verlag Berlin Heidelberg 2016

\begin{abstract}
Aims/hypothesis The aim of this study was to investigate the effects of exercise, vs rest, on circulating insulin and glucose, following pre-exercise insulin pump basal rate reduction.

Methods This was an open-label, two-stage randomised crossover study of 14 adults (seven women, seven men) with type 1 diabetes established on insulin pump therapy. In each stage, participants fasted and insulin delivery was halved following a single insulin basal rate overnight. Exercise (30 min moderate-intensity stationary bicycle exercise, starting $60 \mathrm{~min}$ post-basal reduction) and rest stages were undertaken in random order at a university hospital. Randomisation was computer-generated, and allocation concealed via sequentially numbered sealed opaque envelopes. Venous blood was collected at $15 \mathrm{~min}$ intervals from $60 \mathrm{~min}$ pre- until $210 \mathrm{~min}$ post-
\end{abstract}

Electronic supplementary material The online version of this article (doi:10.1007/s00125-016-3981-9) contains peer-reviewed but unedited supplementary material, which is available to authorised users.

David N. O’Neal

dno@unimelb.edu.au

1 Department of Medicine, St Vincent's Hospital, University of Melbourne, 29 Regent Street, Fitzroy, Melbourne, VIC 3065, Australia

2 Department of Endocrinology \& Diabetes, St Vincent's Hospital Melbourne, Melbourne, VIC, Australia

3 Department of Pathology, University of Melbourne, Melbourne, VIC, Australia

4 Department of Cardiology, St Vincent's Hospital Melbourne, Melbourne, VIC, Australia

5 NHMRC Clinical Trials Centre, University of Sydney, Sydney, NSW, Australia

6 Sports Cardiology, Baker IDI Heart and Diabetes Institute, Melbourne, VIC, Australia basal rate reduction. Changes in plasma free insulin (the primary outcome), and changes in plasma glucose, with exercise were compared with changes when resting. Outcomes were assessed blinded to group assignment.

Results Following basal rate reduction when rested, mean $( \pm$ SE) free insulin decreased by $4.9 \pm 2.9 \%, 16.2 \pm 2.6 \%$ and $18.6 \pm 3.2 \%$ at 1,2 and $3 \mathrm{~h}$, respectively $(p<0.05$ after $75 \mathrm{~min}$ ). With exercise, relative to rest, mean free insulin increased by $6 \pm 2 \mathrm{pmol} / \mathrm{l}$ after $15 \mathrm{~min}$ and $5 \pm 2 \mathrm{pmol} / \mathrm{l}$ after $30 \mathrm{~min}(p<0.001)$, then declined post-exercise $(p<0.001)$. Three participants (mean baseline glucose $5.0 \pm 0.1 \mathrm{mmol} / \mathrm{l}$ ) required glucose supplementation to prevent or treat exercise-related hypoglycaemia. In the other 11 participants (mean baseline glucose $8.4 \pm 0.5 \mathrm{mmol} / \mathrm{l}$ ), glucose increased by $0.8 \pm 0.3 \mathrm{mmol} / 1$ with exercise $(p=0.028)$.

Conclusions/interpretation Halving the basal insulin rate $1 \mathrm{~h}$ prior to exercise did not significantly reduce circulating free insulin by exercise commencement. Exercise itself transiently increased insulin levels. In participants with low-normal glucose pre-exercise, hypoglycaemia was not prevented by insulin basal rate reduction alone. Greater insulin basal rate reduction and supplemental carbohydrate may be required to prevent exercise-induced hypoglycaemia.

Trial registration ANZCTR.org.au ACTRN12613000581763 Funding Australian Diabetes Society, Hugh DT Williamson Foundation, Lynne Quayle Charitable Trust Fund

Keywords Exercise $\cdot$ Hypoglycaemia $\cdot$ Insulin pump $\cdot$ Type 1 diabetes

\section{Introduction}

Exercise has health benefits for people with type 1 diabetes, including improved physical fitness, insulin sensitivity, lipid 
profile and blood pressure, which reduce cardiovascular risk [1]. Current ADA recommendations for people with diabetes are for at least $150 \mathrm{~min} /$ week of moderate-intensity aerobic physical activity [2], corresponding to $30 \mathrm{~min} /$ day for 5 days per week. Evidence for improved overall glycaemia with exercise in type 1 diabetes is inconsistent [3, 4], and likely impacted by exercise-related insulin and diet regimens.

Endogenous insulin levels fall with moderate- and high-intensity exercise in people without diabetes [5], maintaining normoglycaemia and enabling mobilisation of carbohydrate and lipid energy reserves for exercising muscle. However, matching subcutaneous insulin delivery to the varying insulin requirements of exercising people with type 1 diabetes remains a therapeutic challenge [6]. Hypoglycaemia risk increases in people with type 1 diabetes during and after exercise via various mechanisms including increased muscle glucose use, inadequate hepatic glucose output, accelerated insulin absorption and increased insulin sensitivity [7-9]. Despite the numerous benefits of exercise, fear of hypoglycaemia has been reported as the strongest barrier to regular physical activity for those with type 1 diabetes [10].

Although carbohydrate ingestion can prevent exerciserelated hypoglycaemia [11, 12], the timing, amount and composition is debated, and additional carbohydrate reduces the weight-related benefits of exercise. Both prior hypoglycaemia and prior exercise impair counter-regulatory responses to hypoglycaemia in type 1 diabetes [13, 14]. Conversely, insufficient insulin during exercise exaggerates the glucagon response, increasing hepatic glucose output and ketogenesis, and amplifies the catecholamine response, thereby impairing glucose use and increasing lipolysis [7]. Further complicating appropriate insulin dosing are the varied glycaemic responses to exercise type (aerobic, resistance or mixed) $[15,16]$.

For people with type 1 diabetes, insulin pump therapy provides the most flexible insulin delivery, may reduce severe hypoglycaemia risk [17, 18], and may be better placed than multiple daily insulin injections to address exercise-related insulin requirements [9, 19]. An observational study of people with type 1 diabetes undertaking aerobic exercise compared those on insulin pump therapy with those using multiple daily injections. Similar glucose levels were reported during exercise, though less hyperglycaemia occurred post-exercise in those treated with insulin pumps [20].

Insulin pump dose adjustment advice for exercise is inconsistent, and based upon limited evidence [19, 21]. Insulin basal rate reductions of 30-100\% - commencing 30-120 min pre-exercise until exercise completion - are generally advised to avoid exercise-related hypoglycaemia [22-25], though their optimal timing and magnitude for various types of exercise are not fully elucidated. Suspension of basal insulin at exercise commencement can reduce hypoglycaemia, though often at the expense of hyperglycaemia post-exercise [19].
Pharmacokinetic profiles of subcutaneously injected rapid-acting insulin analogues suggest 50-55 min to maximum insulin levels $[26,27]$. In contrast, when one of these insulin analogues was delivered via insulin pump, following pump suspension plasma insulin levels fell by $\sim 50 \%, \sim 60 \%$ and $\sim 70 \%$ at 1,2 and $3 \mathrm{~h}$, respectively, with resultant hyperglycaemia $\sim 3 \mathrm{~h}$ post-suspension [28].

We hypothesised first that current health professional practice regarding insulin pump basal rate reduction prior to moderate-intensity aerobic exercise, such as halving basal delivery $1 \mathrm{~h}$ pre-exercise, may not result in a timely reduction in circulating free insulin; and second that exercise itself impacts insulin absorption during pump therapy. The present study aimed to characterise circulating free insulin levels following a $50 \%$ reduction in basal insulin delivery pre-exercise, and to examine the effects of exercise on free insulin and glucose levels.

\section{Methods}

The study was conducted at St Vincent's Hospital Melbourne following approval by the hospital's ethics committee, and undertaken in accordance with the principles of the Declaration of Helsinki. Each participant provided written informed consent. All study visits were conducted by the same two researchers.

\section{Participants}

Participants were aged $>18$ years with type 1 diabetes, able to perform moderate-intensity exercise, and on insulin pump therapy for $\geq 3$ months with established insulin delivery variables including overnight basal rates between 0.50 and $1.40 \mathrm{U} / \mathrm{h}$. To enable implementation of a single basal rate from 02:00 hours on study days, participants were excluded if their usual 02:00-07:00 hours basal rates varied by $>0.2 \mathrm{U} / \mathrm{h}$. Other exclusion criteria were ischaemic heart disease symptoms, abnormal electrocardiogram, cardiac condition precluding exercise, diabetic ketoacidosis or severe hypoglycaemia within the preceding month, pregnancy, renal impairment (estimated GFR $<60 \mathrm{ml}$ $\min ^{-1} 1.73 \mathrm{~m}^{-2}$ ), BMI $<18$ or $>32 \mathrm{~kg} / \mathrm{m}^{2}$, skin condition in area of cannula or device placement, and allergy to insulin aspart.

\section{Experimental protocol}

This was a prospective, open-label, two-stage randomised crossover study (Fig. 1). Following clinical assessment, participants were allocated to exercise and rest stages sequentially in random order, with 1-8 weeks between study stages. Randomisation was performed by computerised sequence generation, and allocation concealed via sequentially numbered sealed opaque envelopes until 


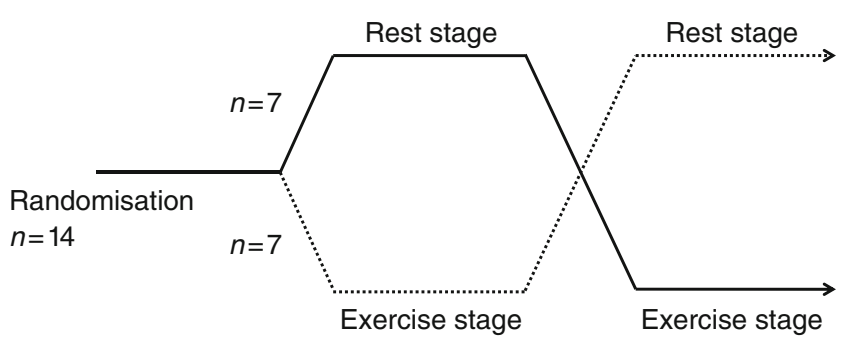

Fig. 1 Randomised crossover study design

enrolment completion. Outcomes were assessed blinded to group assignment.

Insulin basal rate reduction Subcutaneous aspart insulin (NovoRapid, Novo Nordisk, Bagsvaerd, Denmark) was infused as per participants' usual pump settings via standardised equipment: Paradigm Veo insulin pump (Medtronic, Northridge, CA, USA), $9 \mathrm{~mm}$ cannula inserted $90^{\circ}$ to anterior abdomen, $60 \mathrm{~cm}$ infusion line. The insulin basal rate was reduced by $50 \% 1 \mathrm{~h}$ pre-exercise, and at an equivalent time during the rest stage. The magnitude and timing of basal reduction was informed by published literature $[19,21-23]$ and a survey of 17 insulin pump-experienced healthcare professionals in Melbourne, Australia. Those surveyed provided recommendations for a hypothetical patient new to insulin pump therapy undertaking $30 \mathrm{~min}$ of planned moderate-intensity bicycle exercise. Survey results were to advise the patient to institute a temporary basal rate reduction of median (range) $50 \%(0-66 \%)$ starting $60 \mathrm{~min}$ $(0-120 \mathrm{~min})$ pre-exercise until $60 \mathrm{~min}(0-210 \mathrm{~min})$ post-exercise. Only four of 17 clinicians surveyed recommended supplemental carbohydrate pre-exercise.

Rest stage Day 1: Participants attended the temperaturecontrolled clinical trial centre for insulin delivery line change. To achieve a constant overnight basal insulin delivery rate during the study, basal rate adjustments of up to $0.2 \mathrm{U} / \mathrm{h}$ were made if required. For safety, real-time continuous glucose monitoring was implemented using an Enlite glucose sensor with MiniLink transmitter (Medtronic, Northridge, CA, USA) which transmitted glucose levels to the insulin pump every 5 min. Day 2: Participants undertook their usual activities. Day 3: Participants fasted, did not administer insulin boluses after midnight, maintained a constant basal rate from 02:00 hours, and attended the clinical trial centre by 08:00 hours. Bilateral 20 gauge intravenous cubital fossa cannulae were inserted, and intravenous normal saline $(0.9 \% \mathrm{NaCl}[154 \mathrm{mmol} / \mathrm{l}])$ commenced at $100 \mathrm{ml} / \mathrm{h}$. Participants were observed resting for $270 \mathrm{~min}$, with non-arterialised venous samples collected (from the contralateral arm to intravenous fluid infusion) at $15 \mathrm{~min}$ intervals for plasma insulin and glucose, and whole blood meter glucose (Fig. 2). Following 60 min rest, the basal rate was reduced by $50 \%$ for $210 \mathrm{~min}$, after which participants resumed their usual activities and diabetes care.

Exercise stage Study procedures were as for the rest stage, with addition of a standardised exercise protocol on Day 3 commencing 60 min after insulin basal rate reduction (Fig. 2). The exercise was a $30 \mathrm{~min}$ moderate-intensity stationary bicycle protocol, consistent with ADA exercise guidelines [2]. Workload was gradually increased over $5 \mathrm{~min}$ to a target of $65-70 \%$ of age-predicted maximal heart rate [29], followed by $25 \mathrm{~min}$ maintenance of target heart rate with bicycle wattage adjusted by the investigator to maintain the target. Following exercise, participants were monitored at rest for $120 \mathrm{~min}$. Samples were collected as for rest stage Day 3.

Study clinical decisions were based upon meter glucose, which was subsequently confirmed by plasma glucose measurement. Glucose supplementation was administered only in the setting of hypoglycaemia (blood glucose $<3.9 \mathrm{mmol} / \mathrm{l}$ ) or impending hypoglycaemia (blood glucose $<4.4 \mathrm{mmol} / \mathrm{l}$ and falling). The primary study outcome was the impact of exercise compared with rest, following a basal rate reduction, on changes in circulating free insulin levels. Secondary outcomes included the impact of exercise on glucose levels, and on the requirement for glucose supplementation to prevent or treat hypoglycaemia.

\section{Laboratory assays}

Plasma anti-insulin antibodies were measured via an in-house radioimmunoassay, adapted from a published method [30], to determine the proportion of sample bound without anti-serum compared with the assay upper limit of normal. Insulin was quantified by radioimmunoassay, competing with ${ }^{125}$ I-labelled insulin on insulin-specific anti-sera. When antibodies were negative, plasma free insulin was quantified by an in-house insulin assay, adapted from a published method [30]; intra-assay CV $4.1 \%$ at insulin level $39 \mathrm{pmol} / \mathrm{l}$, and $4.4 \%$ at $161 \mathrm{pmol} / \mathrm{l}$. When antibodies were present, plasma was pre-treated with polyethylene glycol to precipitate bound insulin [31], then free insulin was measured by radioimmunoassay using a human insulin-specific radioimmunoassay kit (Millipore, Billerica, MA, USA); intraassay CV $10.6 \%$ at insulin level $38 \mathrm{pmol} / 1$ and $6.1 \%$ at $167 \mathrm{pmol} / \mathrm{l}$. C-peptide was measured by chemiluminescent microparticle immunoassay (Abbott Architect; Abbott Laboratories, Abbott Park, IL, USA), with a lower limit of quantification of $0.03 \mathrm{nmol} / \mathrm{l}$. All insulin samples from each participant were measured in the same assay run.

Laboratory plasma glucose was quantified using the glucose oxidase method [32] (YSI 2300 STAT Plus Glucose Analyzer; YSI Life Sciences, Yellow Springs, OH, USA); assay CV $2.4 \%$ at $4.4 \mathrm{mmol} / \mathrm{l}$ and $2.9 \%$ at $26.0 \mathrm{mmol} / \mathrm{l}$.

Meter glucose was measured by Optium Xceed (Abbott Diabetes Care, Whitney, UK) using the glucose oxidase method with whole venous blood. This meter meets the 
Fig. 2 Day 3 protocol

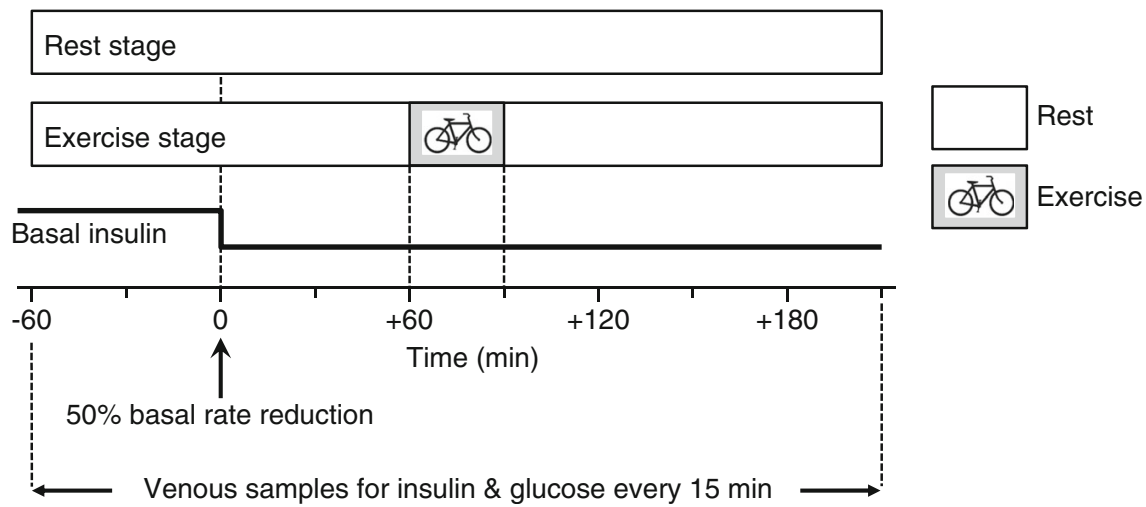

International Organization for Standardization 15197:2013 standard [33].

\section{Statistical analysis}

Results are presented as mean $\pm \mathrm{SE}$, unless otherwise specified. This was an exploratory study, with an absence of available data allowing calculations of statistical power. Although there is information available regarding pharmacokinetic and pharmacodynamic variability of insulin analogue injections [34], the authors are not aware of published data regarding intra-individual variability of insulin analogue absorption when infused subcutaneously via insulin pump. The variability seen with insulin injections may not translate to basal infusion via insulin pump.

Statistical analyses used Stata Version 13.0 (StataCorp, College Station, TX, USA). Analyses included descriptive statistics, Student's paired $t$ tests, and repeated measures ANOVA to compare plasma insulin and glucose between study stages, and to evaluate changes after insulin basal rate reduction and with exercise relative to rest. The significance of factors was estimated using the underlying regression model after performing ANOVA. The impact of multiple comparisons was accounted for by using the Bonferroni correction method. Two-tailed $p$ values $<0.05$ were considered statistically significant. Figures were created using GraphPad Prism version 6.05 (GraphPad, La Jolla, CA, USA).

\section{Results}

Fourteen participants undertook and completed the study protocol between May 2013 and March 2014. Participant clinical characteristics are summarised in Table 1. All participants, except for one with a physically demanding occupation, engaged in regular leisure time physical activity. Six participants (43\%) performed less than the recommended amount of exercise (i.e. $<150 \mathrm{~min} /$ week of moderate-intensity exercise), and three participants (21\%) were involved in regular exercise for 1-2 $\mathrm{h} /$ day with a goal of maintaining a high level of fitness. All participants had fasting C-peptide levels $<0.1 \mathrm{nmol} / \mathrm{l}$.

\section{Changes in circulating free insulin levels}

Changes in circulating free insulin levels are presented by study stage in Fig. 3, and untransformed free insulin levels are presented in electronic supplementary material (ESM) Fig. 1. There was no difference in mean baseline plasma insulin between rest and exercise stages $(58 \pm 3 \mathrm{pmol} / 1$ vs $58 \pm 2 \mathrm{pmol} / \mathrm{l} ; p=0.94)$. Insulin levels were at steady state during the observation hour pre-basal rate reduction in both stages. Following insulin basal rate reduction, when participants remained rested mean circulating free insulin levels decreased from baseline by $4.9 \pm 2.9 \%$, $16.2 \pm 2.6 \%$ and $18.6 \pm 3.2 \%$ at 1,2 and $3 \mathrm{~h}$, respectively, with the difference in levels from baseline reaching statistical significance 75 min post-basal rate reduction. Anti-insulin antibody

Table 1 Clinical characteristics of study participants

\begin{tabular}{|c|c|}
\hline Characteristic & $n=14$ \\
\hline Age (years) & $42 \pm 10$ \\
\hline Sex, female & $7(50 \%)$ \\
\hline BMI $\left(\mathrm{kg} / \mathrm{m}^{2}\right)$ & $25.8 \pm 4.0$ \\
\hline Duration of diabetes (years) & $21 \pm 9$ \\
\hline Duration of insulin pump therapy (years) & $4.8 \pm 3.4$ \\
\hline $\mathrm{HbA}_{1 \mathrm{c}}(\%)$ & $7.2 \pm 0.5$ \\
\hline $\mathrm{HbA}_{1 \mathrm{c}}(\mathrm{mmol} / \mathrm{mol})$ & $55 \pm 5$ \\
\hline Estimated GFR ( $\left.\mathrm{ml} \mathrm{min} \min ^{-1} 1.73 \mathrm{~m}^{-2}\right)$ & $99 \pm 14$ \\
\hline Microvascular diabetes complications & $2(14 \%)$ \\
\hline Macrovascular diabetes complications & $6(43 \%)$ \\
\hline Total daily insulin $\left(\mathrm{U} \mathrm{kg}^{-1}\right.$ day $\left.^{-1}\right)$ & $0.55 \pm 0.10$ \\
\hline Basal insulin proportion of total (\%) & $46.0 \pm 10.2$ \\
\hline Overnight basal insulin rate (U/h) & $0.83 \pm 0.26$ \\
\hline Insulin antibody positive & $11(79 \%)$ \\
\hline \multicolumn{2}{|l|}{ Daily physical activity } \\
\hline$<30 \min |30-60 \min |>60 \min$ & $6(43 \%)|5(36 \%)| 3(21 \%$ \\
\hline
\end{tabular}

Continuous data are presented as mean $\pm \mathrm{SD}$. Categorical data are presented as frequency (\%) 


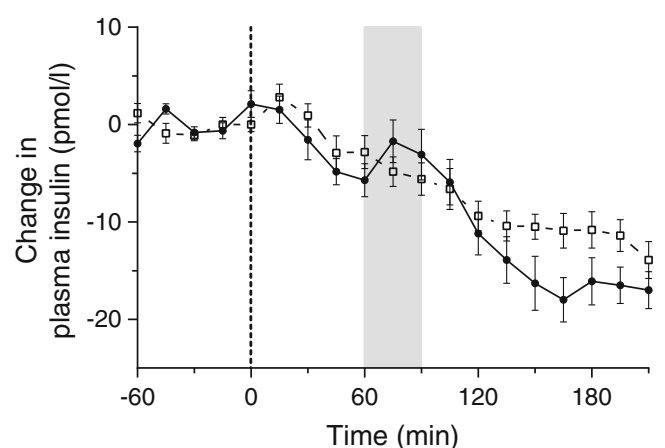

Fig. 3 Impact of basal rate reduction at $0 \mathrm{~min}$, and exercise (exercise stage only, represented by shaded box), on change in circulating free insulin from baseline. Profiles by study stage: rest, white squares; exercise, black circles. Values are mean $\pm \mathrm{SE}$

status did not influence the rate of decline in circulating insulin post-basal rate reduction $(p=0.49)$.

During the hour after basal rate reduction, in the rest stage the mean insulin level went from baseline $58 \pm 3 \mathrm{pmol} / \mathrm{l}$ to $55 \pm 2 \mathrm{pmol} / \mathrm{l}$. During the corresponding hour in the exercise stage (pre-exercise) the insulin level went from baseline $58 \pm 2 \mathrm{pmol} / 1$ to $52 \pm 2 \mathrm{pmol} / \mathrm{l}$. Insulin levels diverged between study stages after exercise onset. Mean free insulin levels increased with exercise, relative to remaining at rest, by $6 \pm 2 \mathrm{pmol} / \mathrm{l}$ after $15 \mathrm{~min}$, and by $5 \pm 2 \mathrm{pmol} / \mathrm{l}$ after $30 \mathrm{~min}$ of exercise $(p<0.001)$. Comparing study stages, insulin levels were lower during the $2 \mathrm{~h}$ post-exercise completion than the corresponding interval without exercise $(p<0.001)$. Of note, the hour following exercise completion was characterised by an accelerated fall in circulating insulin $(-13 \pm 2 \mathrm{pmol} / \mathrm{l}$ post-exercise vs $-5 \pm 1 \mathrm{pmol} / 1$ with rest; $p=0.007)$. The amount of insulin delivered was identical in both study stages, and plasma insulin AUC was only significantly different between the stages post-exercise (ESM Table 1).

\section{Changes in glucose levels}

During the $12 \mathrm{~h}$ prior to basal rate reduction, in rest vs exercise stages there were 12 vs 9 episodes of

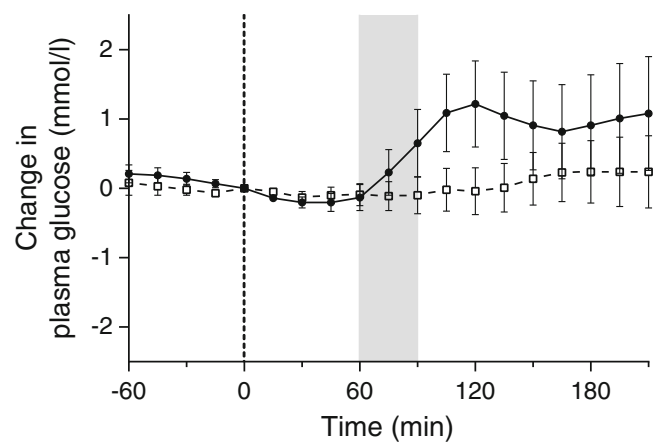

Fig. 4 Impact of basal rate reduction at $0 \mathrm{~min}$, and exercise (exercise stage only, represented by shaded box), on change in plasma glucose from time of basal rate reduction. Profiles by study stage: rest, white squares; exercise, black circles. Values are mean $\pm \mathrm{SE}$ hyperglycaemia ( $>10.0 \mathrm{mmol} / \mathrm{l})$, and seven vs six episodes of hypoglycaemia $(<3.9 \mathrm{mmol} / \mathrm{l})$, respectively. There was no severe hypoglycaemia. These episodes did not impact insulin dosing after 02:00 hours (i.e. no insulin boluses or basal adjustments).

During the exercise stage, three participants $(21 \%)$ required glucose supplementation to prevent or treat hypoglycaemia. One participant had plasma glucose $<3.9 \mathrm{mmol} / \mathrm{l}$ with symptomatic hypoglycaemia during exercise, reaching glucose nadir $3.2 \mathrm{mmol} / \mathrm{l}$, and was treated with $30 \mathrm{~g}$ oral glucose then intravenous $5 \%$ dextrose infusion $(100 \mathrm{ml} / \mathrm{h})$ until study completion. To maintain normoglycaemia during exercise, two other participants required intravenous $5 \%$ dextrose $(100 \mathrm{ml} / \mathrm{h})$, which was commenced $90 \mathrm{~min}$ and $45 \mathrm{~min}$ pre-exercise, respectively, and continued until study completion. Treatment decisions were based upon clinical urgency when meter glucose was $<4.4 \mathrm{mmol} / \mathrm{l}$ and predicted to reach $<2.8 \mathrm{mmol} / \mathrm{l}$ within $30 \mathrm{~min}$ without glucose intervention, which exercise may have exacerbated. The mean baseline glucose of these three participants requiring glucose supplementation was $5.0 \pm 0.1 \mathrm{mmol} / 1$, which was significantly lower than the mean baseline glucose of those who did not require glucose administration during or after exercise $(8.4 \pm 0.5 \mathrm{mmol} / 1 ; p=0.004)$. During the rest stage one participant required glucose supplementation to maintain normoglycaemia, and was treated with intravenous $5 \%$ dextrose $(100 \mathrm{ml} / \mathrm{h})$ from $45 \mathrm{~min}$ pre-basal rate reduction until study completion. Insulin delivery did not deviate from the protocol in any participant. There was no hypoglycaemia during the 120 min of observation post-exercise for any participant.

The three participants who received glucose supplementation (one in both study stages) were excluded from the following analysis of changes in glucose levels.

Mean baseline plasma glucose in the rest stage was $9.4 \pm 0.5 \mathrm{mmol} / \mathrm{l}$, and in the exercise stage was $8.4 \pm 0.5$ $\mathrm{mmol} / 1$ ( $p=0.21$, ESM Fig. 2). Plasma glucose levels were at steady state prior to basal rate reduction in each study stage. With rest, following the $50 \%$ basal rate reduction there was no significant change in plasma glucose during $210 \mathrm{~min}$ of observation ( $p=0.86$, Fig. 4). Plasma glucose levels increased with exercise and the increase was sustained post-exercise $(p<0.001)$. Relative to rest, plasma glucose increased by $0.8 \pm 0.3 \mathrm{mmol} / 1$ after $30 \mathrm{~min}$ exercise $(p=0.028)$. The maximum mean glucose increment in the exercise stage was $1.2 \pm 0.4 \mathrm{mmol} / \mathrm{l}$, occurring $30 \mathrm{~min}$ post-exercise completion.

\section{Discussion}

Current expert guidelines for people with type 1 diabetes using insulin pump therapy recommend a pre-exercise reduction in insulin basal rate to minimise hypoglycaemia risk 
[22-24]. In this study of adult insulin pump users undertaking a commonly recommended 30 min moderate-intensity aerobic exercise regimen, we tested these guidelines.

\section{Main findings}

Principal study findings are: post-implementation of a basal rate reduction congruent with clinical guidelines, there was a gradual decline in circulating free insulin which did not mirror the reduction in insulin delivery; that a $50 \%$ reduction in basal insulin delivery did not prevent hypoglycaemia when pre-exercise glucose levels were low-normal; and paradoxically, despite an insulin basal rate reduction implemented pre-exercise, moderate-intensity aerobic exercise transiently increased circulating free insulin.

We observed a decrease in circulating free insulin after the $50 \%$ basal rate reduction when participants remained resting of approximately $5 \%, 16 \%$ and $19 \%$ at 1,2 and $3 \mathrm{~h}$, respectively. There was a modest exercise-related insulin level increase, followed by an accelerated decline in circulating free insulin post-exercise, relative to the non-exercise setting. We hypothesise that the observed exercise-related increase in circulating free insulin may be due to increased cardiac output and increased cutaneous blood flow associated with thermoregulation during exercise promoting subcutaneous insulin absorption. The subsequent accelerated decline could be consistent with exercise-related depletion of the subcutaneous insulin reservoir, and mechanical factors such as body movement and/or pressure may also contribute. Altered renal insulin clearance mediated by exercise-related decreases in renal blood flow may also influence circulating free insulin levels.

With regard to changes in glycaemia following a $50 \%$ reduction in basal insulin delivery with participants remaining at rest, we observed unchanged glucose levels during $3.5 \mathrm{~h}$. In contrast, with exercise, glucose levels rose in participants with baseline glucose $>5.5 \mathrm{mmol} / \mathrm{l}$. Of note, this glucose increase paradoxically occurred despite increased circulating insulin with exercise, raising the untested hypothesis of counter-regulatory hormones influencing circulating glucose levels. This plasma glucose rise was sustained during recovery. The accelerated reduction in circulating free insulin levels post-exercise may have contributed to maintaining these elevated glucose levels.

For the majority of participants with glucose in the mid- to high-normal range, our study's $50 \%$ basal rate reduction pre-exercise, together with the physiological responses to exercise, was sufficient to prevent hypoglycaemia. However, in three participants $(21 \%)$ with low-normal glucose levels at exercise commencement, the $50 \%$ basal rate reduction alone was insufficient to prevent hypoglycaemia.

\section{Previous studies}

Previous type 1 diabetes exercise studies have investigated non-analogue insulin therapy, insulin injections, unplanned exercise, or focused on glucose levels alone [19, 21, 34-38]. The insulin level fall observed in our study was significantly less than that observed in the pump study by Attia et al where insulin delivery was ceased [28]. The modest increase in circulating free insulin associated with moderate-intensity aerobic exercise which we identified is consistent with insulin analogue findings by Admon et al in a paediatric cohort [21], though contrasts findings by Mitchell et al from the pre-analogue insulin era [35]. This may relate to more rapid absorption of analogue insulin than human or animal insulins.

While we recognise the differences between aerobic and anaerobic exercise, our observations in adults are consistent with publications relating to high-intensity sprint exercise increasing glucose levels in adults on insulin pumps [35]. In contrast, published paediatric glycaemic data where insulin delivery was reduced or ceased at the time exercise commenced reported blood glucose reductions with exercise $[19,21]$. This may be explained by differences in the timing of the reduction in insulin delivery, duration and intensity of the exercise and the study participant characteristics.

\section{Strengths and limitations}

Novel findings of the present study relate to the impact of changes in basal analogue insulin delivery, and of exercise itself, on insulin and glucose levels in adults undertaking planned exercise. The changes in basal insulin delivery implemented by the present study protocol are clinically relevant as they are congruent with current guidelines. Therefore, these findings have direct applicability to clinical practice. The study design was robust. Insulin, insulin pumps and infusion lines were uniform. In addition, the protocol incorporated a fixed basal insulin infusion rate overnight, and synchronous identical basal rate reductions in each study stage, in the absence of food or bolus insulin, to achieve steady-state circulating free insulin levels at baseline. By implementing the protocol in the fasting state, we standardised insulin delivery by avoiding boluses characterised by differing insulin:carbohydrate ratios and insulin sensitivity factors. Such variability would have been superimposed on the impact of the basal rate change, and elimination of that potential heterogeneity facilitated interpretation of results in this small cohort.

There are study limitations. The standardised exercise protocol does not enable dissection of the relative effects of exercise duration and intensity. Studies with longer duration of observation post-exercise have demonstrated that late-onset 
hypoglycaemia may occur many hours post-aerobic exercise, often overnight, related to muscle glycogen repletion and increased insulin sensitivity $[9,39]$. As we did not follow participants longer than $2 \mathrm{~h}$ post-exercise, we cannot comment on late-onset hypoglycaemia. Furthermore, only adults were studied and caution is recommended in extrapolating results to a paediatric population. Finally, counter-regulatory hormone levels were not measured, precluding assessment of their contribution to glucose changes.

\section{Clinical implications}

Our findings reinforce current clinical recommendations for supplemental carbohydrate pre-exercise when blood glucose levels are relatively low [24]. Therefore, when glucose is $\leq 7 \mathrm{mmol} / \mathrm{l}$ prior to exercise, to minimise hypoglycaemia risk we recommend pre-exercise supplementary carbohydrate ingestion and to consider a basal insulin reduction greater than $50 \%$. An extended period of observation post-exercise will be relevant to future research aiming to optimise insulin delivery for exercise. In addition, based on these results we suggest that to achieve clinically relevant reductions in circulating free insulin for exercise, insulin basal rate reductions between $50 \%$ and $100 \%$ may be required. We recognise that implementing basal rate reductions earlier than $1 \mathrm{~h}$ pre-exercise may pose practical challenges for patients as often exercise is unplanned. When basal rate reductions are implemented immediately pre-exercise, larger basal rate reductions, with their more rapid effect on circulating insulin, may be required.

For those participants with pre-exercise glucose $>7 \mathrm{mmol} / \mathrm{l}$, the impact of the $50 \%$ basal reduction $1 \mathrm{~h}$ pre-exercise on glycaemia at the time of, and immediately following, exercise was minimal. This was underlined by the minor increase in glucose with exercise in the face of the observed increase in circulating insulin. It could therefore be argued that in these situations the major factor preventing hypoglycaemia during and immediately following exercise is the counter-regulatory hormone response. In individuals with pre-exercise glucose $>7 \mathrm{mmol} / \mathrm{l}$, basal insulin reductions greater than $50 \%$ may be counterproductive. However, the $50 \%$ reduction implemented in the present study may represent the most appropriate option as it resulted in only a minor, clinically acceptable, rise in glucose during exercise, and potentially could diminish the risk of post-exercise hypoglycaemia.

Our findings extend beyond exercise. The absence of a rise in glucose levels $3.5 \mathrm{~h}$ after $50 \%$ basal rate reduction may have clinical relevance in the routine management of people with type 1 diabetes when determining insulin pump settings. For example, basal insulin delivery profiles characterised by frequent minor basal rate changes may not represent optimal clinical care.

\section{Future research}

Our findings suggest further research elucidating changes in circulating insulin following alterations in basal delivery prior to exercise is warranted. Effects of basal rate reductions between $50 \%$ and $100 \%$ remain to be explored. In addition, the observed exercise-related modest insulin level rise requires further investigation into factors potentially affecting absorption from the subcutaneous insulin depot, and impacting renal clearance of insulin with exercise. The impact of differing exercise types and intensities upon circulating free insulin levels merits research. Finally, we did not measure the contribution of counter-regulatory hormones to glycaemic fluctuations with exercise, and exploration of these hormonal responses in future studies would provide additional insights.

\section{Conclusions}

The present study provides insights into the effects of a commonly recommended insulin pump regimen for aerobic exercise on circulating free insulin and glucose levels. The results address a gap in the literature and may help inform clinical practice. Although insulin pumps have the capability to precisely and flexibly vary basal insulin delivery into subcutaneous tissue, the results of the present study suggest that this precision in delivery is not reflected in circulating insulin levels and action. Decreasing insulin pump dosing pre-exercise according to current type 1 diabetes practice guidelines did not significantly reduce circulating free insulin levels during exercise. A concerning minority remained at risk of hypoglycaemia. This suggests that for people with type 1 diabetes with pre-exercise glucose levels in the lower half of the target range, reductions in basal insulin delivery pre-exercise should be greater than current guidelines, and be accompanied by supplemental carbohydrate, in order to prevent early exercise-induced hypoglycaemia.

Acknowledgements The authors thank all the volunteers for their participation and enthusiasm. We also thank Medtronic for providing glucose sensing materials.

Funding This project was funded by grants from the Australian Diabetes Society ADS-Sanofi Diabetes Research Grant programme, the Medical Research \& Technology in Victoria programme supported by The Hugh DT Williamson Foundation, and the Lynne Quayle Charitable Trust Fund managed by Equity Trustees. SAM is supported by a University of Melbourne postgraduate scholarship. ALG is supported by a Career Development Scholarship from the National Health and Medical Research Council and a Future Leaders Fellowship from the National Heart Foundation of Australia. AJJ is supported by a Sydney Medical School Fellowship from the University of Sydney.

No funding source had a role in the study design or conduct; collection, management, analysis, or interpretation of the data; or preparation, review, or approval of the manuscript. 
Duality of interest The non-profit employers of the authors have received grant support from Medtronic for other investigator-initiated studies. AJJ and DNO have previously received honoraria from Medtronic. All other authors declare that there is no duality of interest associated with their contribution to this manuscript.

Contribution statement SAM was involved in all aspects of the study, and wrote the first draft of the manuscript. JCH and JLG contributed to acquisition of data. GMW provided laboratory expertise, and contributed to the analysis and interpretation of data. ALG contributed to study design, and interpretation of data. AJJ and RJM contributed to study conception and interpretation of data. DNO contributed to study conception and design, acquisition of data, and interpretation of data. All authors contributed to revising the manuscript critically and approved the final version. SAM and DNO are the guarantors of this work.

\section{References}

1. Chimen M, Kennedy A, Nirantharakumar K, Pang TT, Andrews R, Narendran P (2012) What are the health benefits of physical activity in type 1 diabetes mellitus? A literature review. Diabetologia 55: 542-551

2. American Diabetes Association (2016) Standards of Medical Care in Diabetes - 2016. Diabetes Care 39(Suppl):S1-S112

3. Quirk H, Blake H, Tennyson R, Randell TL, Glazebrook C (2014) Physical activity interventions in children and young people with Type 1 diabetes mellitus: a systematic review with meta-analysis. Diabet Med 31:1163-1173

4. Kennedy A, Nirantharakumar K, Chimen M et al (2013) Does exercise improve glycaemic control in type 1 diabetes? A systematic review and meta-analysis. PLoS One 8:e58861

5. Petersen KF, Price TB, Bergeron R (2004) Regulation of net hepatic glycogenolysis and gluconeogenesis during exercise: impact of type 1 diabetes. J Clin Endocrinol Metab 89:4656-4664

6. Colberg SR (2013) Exercise and diabetes: a clinician's guide to prescribing physical activity. American Diabetes Association, Alexandria

7. Wasserman DH, Zinman B (1994) Exercise in individuals with IDDM. Diabetes Care 17:924-937

8. Zinman B, Murray FT, Vranic M et al (1977) Glucoregulation during moderate exercise in insulin treated diabetics. J Clin Endocrinol Metab 45:641-652

9. McMahon SK, Ferreira LD, Ratnam N et al (2007) Glucose requirements to maintain euglycemia after moderate-intensity afternoon exercise in adolescents with type 1 diabetes are increased in a biphasic manner. J Clin Endocrinol Metab 92:963-968

10. Brazeau AS, Rabasa-Lhoret R, Strychar I, Mircescu H (2008) Barriers to physical activity among patients with type 1 diabetes. Diabetes Care 31:2108-2109

11. Dubé MC, Weisnagel SJ, Prud'homme D, Lavoie C (2005) Exercise and newer insulins: how much glucose supplement to avoid hypoglycemia? Med Sci Sports Exerc 37:1276-1282

12. Hernandez JM, Moccia T, Fluckey JD, Ulbrecht JS, Farrell PA (2000) Fluid snacks to help persons with type 1 diabetes avoid late onset postexercise hypoglycemia. Med Sci Sports Exerc 32: 904-910

13. Galassetti P, Tate D, Neill RA, Morrey S, Wasserman DH, Davis SN (2003) Effect of antecedent hypoglycemia on counterregulatory responses to subsequent euglycemic exercise in type 1 diabetes. Diabetes 52:1761-1769
14. Sandoval DA, Guy DL, Richardson MA, Ertl AC, Davis SN (2004) Effects of low and moderate antecedent exercise on counterregulatory responses to subsequent hypoglycemia in type 1 diabetes. Diabetes 53:1798-1806

15. Yardley JE, Kenny GP, Perkins BA et al (2013) Resistance versus aerobic exercise: acute effects on glycemia in type 1 diabetes. Diabetes Care 36:537-542

16. Yardley JE, Kenny GP, Perkins BA et al (2012) Effects of performing resistance exercise before versus after aerobic exercise on glycemia in type 1 diabetes. Diabetes Care 35:669-675

17. Misso ML, Egberts KJ, Page M, O’Connor D, Shaw J (2010) Continuous subcutaneous insulin infusion (CSII) versus multiple insulin injections for type 1 diabetes mellitus. Cochrane Database Syst Rev, Issue 1, Art. no.: CD005103. doi: 10.1002/14651858. CD005103.pub2

18. Pickup JC, Sutton AJ (2008) Severe hypoglycaemia and glycaemic control in type 1 diabetes: meta-analysis of multiple daily insulin injections compared with continuous subcutaneous insulin infusion. Diabet Med 25:765-774

19. Diabetes Research in Children Network (DirecNet) Study Group (2006) Prevention of hypoglycemia during exercise in children with type 1 diabetes by suspending basal insulin. Diabetes Care 29: 2200-2204

20. Yardley JE, Iscoe KE, Sigal RJ, Kenny GP, Perkins BA, Riddell MC (2013) Insulin pump therapy is associated with less postexercise hyperglycemia than multiple daily injections: an observational study of physically active type 1 diabetes patients. Diabetes Technol Ther 15:84-88

21. Admon G, Weinstein Y, Falk B et al (2005) Exercise with and without an insulin pump among children and adolescents with type 1 diabetes mellitus. Pediatrics 116:e348-355

22. Lumb AN (2012) The role of newer technologies (CSII and CGM) and novel strategies in the management of type 1 diabetes for sport and exercise. In: Gallen I (ed) Type 1 diabetes. Springer, London, pp 101-113

23. Craig ME, Twigg SM, Donaghue KC et al (2011) National evidence-based clinical care guidelines for type 1 diabetes in children, adolescents and adults. Australian Government Department of Health and Ageing, Canberra

24. Gallen IW (2014) Exercise for people with type 1 diabetes. Med Sport Sci 60:141-153

25. Maahs DM, Horton LA, Chase HP (2010) The use of insulin pumps in youth with type 1 diabetes. Diabetes Technol Ther 12(Suppl): S59-S65

26. Howey DC, Bowsher RR, Brunelle RL, Woodworth JR (1994) [Lys(B28), Pro(B29)]-human insulin. A rapidly absorbed analogue of human insulin. Diabetes 43:396-402

27. Mudaliar SR, Lindberg FA, Joyce M et al (1999) Insulin aspart (B28 asp-insulin): a fast-acting analog of human insulin. Absorption kinetics and action profile compared with regular human insulin in healthy nondiabetic subjects. Diabetes Care 22 : $1501-1506$

28. Attia N, Jones TW, Holcombe J, Tamborlane WV (1998) Comparison of human regular and lispro insulins after interruption of continuous subcutaneous insulin infusion and in the treatment of acutely decompensated IDDM. Diabetes Care 21:817-821

29. Tanaka H, Monahan KD, Seals DR (2001) Age-predicted maximal heart rate revisited. J Am Coll Cardiol 37:153-156

30. Albano JD, Ekins RP, Maritz G, Turner RC (1972) A sensitive, precise radioimmunoassay of serum insulin relying on charcoal separation of bound and free hormone moieties. Acta Endocrinol (Copenh) 70:487-509

31. Nakagawa S, Nakayama H, Sasaki T, Yoshino K, Yu YY (1973) A simple method for the determination of serum free insulin levels in insulin-treated patients. Diabetes 22:590-600 
32. Chua KS, Tan IK (1978) Plasma glucose measurement with the Yellow Springs Glucose Analyzer. Clin Chem 24:150-152

33. ISO 15197:2013 (2013) In vitro diagnostic test systems Requirements for blood-glucose monitoring systems for self-testing in managing diabetes mellitus. ISO, Geneva

34. Heinemann L, Weyer C, Rauhaus M, Heinrichs S, Heise T (1998) Variability of the metabolic effect of soluble insulin and the rapid-acting insulin analog insulin aspart. Diabetes Care 21: 1910-1914

35. Mitchell TH, Abraham G, Schiffrin A, Leiter LA, Marliss EB (1988) Hyperglycemia after intense exercise in IDDM subjects during continuous subcutaneous insulin infusion. Diabetes Care 11:311-317

36. Sonnenberg GE, Kemmer FW, Berger M (1990) Exercise in type 1 (insulin-dependent) diabetic patients treated with continuous subcutaneous insulin infusion. Prevention of exercise induced hypoglycaemia. Diabetologia 33:696-703

37. Guelfi KJ, Jones TW, Fournier PA (2005) The decline in blood glucose levels is less with intermittent high-intensity compared with moderate exercise in individuals with type 1 diabetes. Diabetes Care 28:1289-1294

38. Campbell MD, Walker M, Bracken RM et al (2015) Insulin therapy and dietary adjustments to normalize glycemia and prevent nocturnal hypoglycemia after evening exercise in type 1 diabetes: a randomized controlled trial. BMJ Open Diabetes Res Care 3: $\mathrm{e} 000085$

39. Diabetes Research in Children Network (DirecNet) Study Group (2005) Impact of exercise on overnight glycemic control in children with type 1 diabetes mellitus. J Pediatr 147:528-534 